Pesq. Vet. Bras. 34(Supl.1):34-38, dezembro 2014

DOI: $10.1590 / \mathrm{S} 0100-736 \mathrm{X} 2014001300007$

\title{
Fatores anatomofisiologicos que afetam a qualidade oocitária em bovinos ${ }^{1}$
}

\author{
Vanessa M. Chagas², Madson A. Vidal e Silva ${ }^{3}$, José H. Martins ${ }^{3}$, Cícero S. Santos ${ }^{3}$, \\ Hans M.V.S.B. Aguiar ${ }^{2}$, Celso H.S.C Barros ${ }^{2}$, Ricardo M. Chaves ${ }^{3}$ \\ e José R.S. Torres-Júnior ${ }^{2 *}$
}

\begin{abstract}
Chagas V.M., Vidal e Silva M.A., Martins J.H., Santos C.S., Aguiar H.M.V.S.B., Barros C.H.S.C., Chaves R.M. \& Torres Júnior J.R.S. 2014. [Anatomophysiologic factors affecting oocyte quality in cattle.] Fatores anatomofisiológicos que afetam a qualidade oocitária em bovinos. Pesquisa Veterinária Brasileira 34(Supl.1):34-38. Centro de Ciências Agrárias e Ambientais, Universidade Federal do Maranhão, BR-222 Km 4, Chapadinha, MA 65500-000, Brazil. E-mail: jrtorresjr@gmail.com

To study the anatomical and physiological factors that affect the bovine cumulus-oocyte complexes (COCs) quality, were examined 396 ovaries obtained after slaughter of 198 Bos indicus females. Ovaries were categorized in nulliparous vs multiparous and progesterone (P4 - a corpus luteum at least one ovary) vs without progesterone (NP4 - absence of corpus luteum). All follicles were measured and categorized into small $(<6 \mathrm{~mm})$, medium (6 to $9 \mathrm{~mm}$ ) or large $(>9 \mathrm{~mm})$. Then all follicles were punctured and COCs recovered and assessed morphologically. There was no difference in COCs recovery rate or quality from nulliparous $v s$ multiparous females. The rate of denuded or degenerated oocytes was higher in NP4 and expanded COCs were higher in P4. The COCs recovery and grades I and II rates were similar in P4 vs NP4 groups. Small follicles have lower COCs recovery rate than medium or large follicles, but the grade I oocytes rates was higher in small and medium follicles. In conclusion, the category of donor female and progesterone did not affect the quality of COCs selected for PIV and smaller follicles present best quality of COCs.
\end{abstract}

INDEX TERMS: Cumulus-oocyte complex, follicles, OPU, progesterone, cattle.

RESUMO.- Para estudar os fatores anatomofisiológicos que interferem na qualidade de complexos cumulus-oócitos (CCOs) bovinos, foram obtidas 396 ovários após abate de 198 fêmeas Bos indicus em frigorífico. Os ovários foram separados por categorias, sendo distribuídos em nulípara vs multípara e com progesterona (P4 - presença de corpo lúteo em um dos ovários) vs sem progesterona (NP4 - ausência de corpo lúteo). Todos os folículos foram mensurados e categorizados em pequenos $(<6 \mathrm{~mm})$, médios ( 6 a $9 \mathrm{~mm})$ ou grandes $(>9 \mathrm{~mm})$. Em seguida todos os folículos foram puncionados e os CCOs recuperados e avaliados morfologicamente. Não houve diferença na taxa de recuperação nem

\footnotetext{
${ }^{1}$ Recebido em 6 de junho de 2014.

Aceito para publicação em 13 de dezembro de 2014.

${ }^{2}$ Centro de Ciências Agrárias e Ambientais (CCAA), Universidade Federal do Maranhão (UFMA), BR-222 Km 4 s/n, Chapadinha, MA 65500-000, Brasil. *Autor correspondência: jrtorresjr@gmail.com

${ }^{3}$ Centro de Ciências Agrárias (CCA), Universidade Estadual do Maranhão (UEMA), Cidade Universitária Paulo VI, Cx. Postal 9, São Luís, MA 65500-000, Brasil.
}

na qualidade dos CCOs de fêmeas nulíparas vs multíparas. 0 percentual de CCOs desnudos/degenerados foi maior no grupo NP4 e os CCOs expandidos foram superiores no grupo P4. A taxa de recuperação e o percentual de CCOs selecionados para PIV (graus I e II) foram similares nos grupos P4 vs NP4. Folículos pequenos apresentam menor taxa de recuperação em comparação aos de tamanho médio e grande, porém o percentual de CCOs de grau I foi superior em folículos pequenos e médios. Diante dos resultados aqui encontrados conclui-se que a categoria da doadora e a progesterona não influenciaram a qualidade de CCOs selecionados para PIV e que folículos menores apresentam de CCOs de melhor qualidade.

TERMOS DE INDEXAÇÃO: Complexos cúmulus-oócitos, folículos, OPU, progesterona, bovinos.

\section{INTRODUÇÃO}

O Brasil possui o maior rebanho comercial bovino do mundo, e é também o maior produtor mundial de embriões pro- 
duzidos in vitro (PIV), sendo os animais Bos indicus especialmente explorados neste contexto (Rubin 2006).

A produção global de embriões PIV teve aumento pelo sexto ano consecutivo em 2011, com mais de 379 mil embriões transferidos. Somente o Brasil foi responsável por 86\% deste efetivo (IETS 2013, Stroud 2012). Esta liderança nacional pode ser atribuída à aplicação comercial da PIV em larga escala, associada ao elevado número de oócitos recuperados por ovum pick-up (OPU) em fêmeas zebuínas, representadas principalmente pela raça Nelore (Pontes et al. 2011).

Em 2011, foram realizadas no Brasil 53.019 sessões de OPU, produzindo uma média de 15 oócitos e seis embriões (Mapletoft 2013). A média atual do desenvolvimento in vitro permanece em torno de $30 \%$ do total de complexos cúmulus-oócitos (CCOs) maturados. Para cada 8 a 10 CCOs colhidos por OPU há uma produção média de dois embriões transferidos em bovinos de raças européias (Galli et al. 2001). Em animais da raça Nelore (Bos indicus), a taxa de desenvolvimento embrionário permanece na faixa média de 20 a $35 \%$ dos oócitos maturados chegam até o estágio de blastocisto (Viana et al. 2010).

Segundo Vassena et al. (2003) a qualidade do oócito é de suma importância nas tecnologias de reprodução assistida. A presença das células do cumulus é benéfica para a obtenção de embriões após a fertilização in vitro (FIV), pois oócitos desnudos tem baixa taxa de mitose/clivagem embrionária (Fatehi et al. 2005). As interações parácrinas e autócrinas entre os CCOs são responsáveis pela regulação do desenvolvimento embrionário inicial (Gopichandran \& Leese 2006). Além disso, inadequações no ambiente in vitro durante a maturação, fertilização ou cultura podem resultar em severas consequências em termos de normalidade dos blastocistos (Lonergan et al. 2003).

0 estudo da morfologia oocitária inclui avaliações da forma, tamanho do espaço perivitelino, presença de vacuolizações, granulações ou inclusões citoplasmáticas, além de anormalidades da zona pelúcida e do corpúsculo polar (Costa, 1994). Ainda, têm sido descritas diferentes características como tamanho e padrão de crescimento folicular, perfil hormonal e status ovariano (Torres-Júnior et al. 2006a 2006b 2008, Gimenes et al. 2007a 2007b). Estes fatores demonstram que pode ser impossível criar condições ideais para obter $100 \%$ de competência dos complexos cumulus-oócitos (CCO`s) (Basso et al. 2006; Blondin \& Sirard 1995).

Diante destas considerações é importante desenvolver estudos que possam indicar as potenciais influências biológicas que interferem na qualidade oocitária, norteando a seleção de fêmeas doadoras e objetivando alcançar melhor resultados nas biotécnicas da reprodução.

\section{MATERIAL E MÉTODOS}

O estudo foi realizado na cidade de São Luís, Estado do Maranhão, Brasil, entre novembro de 2009 e fevereiro de 2010. Foram coletados ovários de 198 fêmeas bovinas (Bos indicus) abatidas no frigorífico municipal. Após o abate das fêmeas suas genitálias foram recolhidas e os ovários direito e esquerdo retirados. Em seguida, os ovários foram acondicionados separadamente por categorias em nulípara vs multípara e; com progesterona (P4; presença de corpo lúteo em pelo menos um dos ovários) vs sem progesterona (NP4; ausência de corpo lúteo em ambos os ovários). Os ovários foram levados para o laboratório de Reprodução Animal da Faculdade de Medicina Veterinária da Universidade Estadual do Maranhão (UEMA).

No laboratório, os ovários foram mantidos em banho-maria a $37^{\circ} \mathrm{C}$ e os folículos devidamente identificados e mensurados com auxílio de um paquímetro. Para efeito de análise, os folículos foram categorizados em pequenos $(<6 \mathrm{~mm})$, médios $(6-9 \mathrm{~mm})$ ou grandes ( $>9 \mathrm{~mm})$. Em seguida todos os folículos com diâmetro > $3 \mathrm{~mm}$ forma puncionados com auxílio de uma agulha descartável 21 gauge (25mm x 8mm; BD Precision Guide ${ }^{\circledR}$, Brasil) conectada a uma seringa descartável de $10 \mathrm{~mL}$. 0 número de folículos efetivamente puncionados também foi aferido para calcular a taxa de recuperação oocitária (número de oócitos recuperados x100/ número de folículos efetivamente puncionados).

Finalizado o procedimento de aspiração, os complexos cumulus-oócitos (CCOs) recuperados foram transferidos para placas de cultivo celular contendo Solução Salina Fosfatada (PBS) a $37^{\circ} \mathrm{C}$ e avaliados morfologicamente com auxílio de um estereomicroscópio com aumento final de 50x.

Os critérios de avaliação utilizados foram o aspecto do citoplasma quanto à cor, homogeneidade e integridade, presença e número de camadas, além do grau de expansão das células do cumulus. As categorias de classificação dos CCOs foram definidas segundo Costa (1994), conforme segue abaixo: Grau I, CCOs compactos com mais de três camadas de células do cumulus e oócito com citoplasma homogêneo; Grau II, CCOs compactos com três ou menos camadas de células do cumulus e oócitos com citoplasma ligeiramente heterogêneo; Parcialmente desnudos, oócitos mostrando completa remoção das células do cumulus em pelos menos 1/3 da zona pelúcida (ZP); Desnudo e/ou degenerado, ausência de células do cumulus na superfície da ZP e/ou vacuolização e retração do citoplasma e; Cumulus expandido, CCOs com a expansão das células do cumulus (Torres-Júnior et al. 2008).

A classificação morfológica dos CCOs foi considerada variável-resposta e analisada de acordo com as categorias anatomofisiológicas (variáveis classificatórias): nulípara vs multípara; P4 vs NP4 e; folículos pequenos vs médios vs grandes. As frequências percentuais de cada classificação de CCOs em diferentes categorias anatomofisiológicas foram analisadas pelo teste de qui-quadrado (Sampaio 2002). O nível de significância para rejeitar H0 (hipótese de nulidade) foi de 5\%, isto é, para um nível de significância menor que 0,05 , considerou-se efeito das variáveis classificatórias e das suas interações.

\section{RESULTADOS}

No Quadro 1 estão demonstradas a taxa de recuperação e a classificação morfológica dos CCOs de acordo com o grau de maturidade sexual, em fêmeas nulíparas e multíparas, não tendo sido observada diferença significativa entre estas respectivas categorias $(P>0,05)$. Também em relação ao status ovariano, em fêmeas que apresentavam P4 e NP4, não houve efeito da progesterona na taxa de recuperação e percentual de CCOs considerados maturáveis e potencialmente selecionados para PIV (graus I e II), porém a quantidade de oócitos parcialmente desnudos, desnudos e degenerados foi significativamente maior no grupo NP4 quando comparado ao grupo P4 (19\% e 14,9\%, respectivamente) $(\mathrm{P}<0,05)$. 0 contrário ocorreu na quantidade de CCOs expandidos que foi maior no grupo $\mathrm{P} 4$ em relação ao grupo NP4 $(10,7 \%$ e $6,3 \%$, respectivamente). 
Quadro 1. Efeitos principais do grau de maturidade sexual e status ovariano da fêmea doadora sobre a taxa de recuperação e qualidade de CCOs

\begin{tabular}{|c|c|c|c|c|}
\hline \multirow[b]{2}{*}{ Variável } & \multicolumn{2}{|c|}{ Grau de maturidade sexual ${ }^{\mathrm{NS}}$} & \multicolumn{2}{|c|}{ Status ovariano } \\
\hline & $\begin{array}{l}\text { Multíparas no./ } \\
\text { total (\%) }\end{array}$ & $\begin{array}{l}\text { Nulíparas no./ } \\
\text { total }(\%)\end{array}$ & $\begin{array}{l}\text { P4 no./ } \\
\text { total (\%) }\end{array}$ & $\begin{array}{l}\text { NP4 no./ } \\
\text { total (\%) }\end{array}$ \\
\hline Taxa Recu & $1329 / 3176(41,8)$ & $706 / 1764(40,0)$ & $1310 / 3145(41,7)$ & $725 / 1795(40,4)$ \\
\hline Grau I & $776 / 1329(57,6)$ & $393 / 706(55,7)$ & $741 / 1310(56,6)$ & $418 / 725(57,7)$ \\
\hline Grau II & $221 / 1329(16,6)$ & $136 / 706(19,3)$ & $234 / 1310(17,9)$ & $123 / 725(17,0)$ \\
\hline $\begin{array}{l}\text { Parcialmente desnudos/ } \\
\text { Desnudos / Degenerados }\end{array}$ & $211 / 1329(15,9)$ & $122 / 706(17,3)$ & $195 / 1310(14,9)^{\mathrm{b}}$ & $138 / 725(19,0)^{\mathrm{a}}$ \\
\hline Expandidos & $131 / 1329(9,9)$ & $55 / 706(7,8)$ & $140 / 1310(10,7)^{\mathrm{a}}$ & $46 / 725(6,3)^{b}$ \\
\hline
\end{tabular}

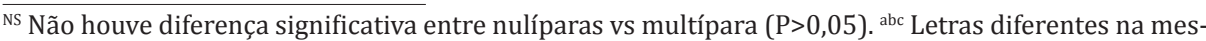
ma linha $(\mathrm{P}<0,05)$ indicam diferença significativa entre $\mathrm{P} 4$ vs NP4; teste de Qui-quadrado. P4 = Fêmea com progesterona (presença de corpo lúteo em pelo menos um dos ovários), NP4 = Fêmea sem progesterona (ausência de corpo lúteo em ambos os ovários).

Quadro 2. Interações entre grau de maturidade sexual e status ovariano da fêmea doadora sobre a taxa de recuperação e qualidade de CCOs

\begin{tabular}{|c|c|c|c|c|}
\hline Variável & $\begin{array}{l}\text { Multípara P4 no. } \\
\text { /total (\%) }\end{array}$ & $\begin{array}{c}\text { Multípara NP4 no. } \\
\text { /total (\%) }\end{array}$ & $\begin{array}{l}\text { Nulípara P4 no. } \\
\text { /total (\%) }\end{array}$ & $\begin{array}{l}\text { Nulípara NP4 no. } \\
\text { /total (\%) }\end{array}$ \\
\hline Taxa Recuperação & $986 / 2247(43,9)^{\mathrm{a}}$ & $343 / 929(36,9)^{\mathrm{b}}$ & $324 / 898(36,1)^{b}$ & $382 / 866(44,1)^{a}$ \\
\hline Grau I & $557 / 986(56,5)$ & $209 / 343(60,9)$ & $184 / 324(56,8)$ & $209 / 382(54,7)$ \\
\hline Grau II & $162 / 986(16,4)^{b}$ & $59 / 343(17,2)^{\mathrm{ab}}$ & $72 / 324(22,2)^{\mathrm{a}}$ & $64 / 382(16,8)^{a b}$ \\
\hline $\begin{array}{l}\text { Parcialmente desnudos/ } \\
\text { Desnudos / Degenerados }\end{array}$ & $152 / 986(15,4)^{b}$ & $59 / 343(17,2)^{a b}$ & $43 / 324(13,3)^{b}$ & $79 / 382(20,7)^{a}$ \\
\hline Expandidos & $115 / 986(11,7)^{\mathrm{a}}$ & $16 / 343(4,7)^{\mathrm{b}}$ & $25 / 324(7,7)^{\mathrm{b}}$ & $30 / 382(7,9)^{\mathrm{b}}$ \\
\hline
\end{tabular}

Quando dissociados os efeitos principais e estudadas as interações entre o grau de maturidade sexual e status ovariano da fêmea doadora, a despeito das diferenças observadas, não se obteve tendência relevante do ponto de vista fisiológico, tanto na taxa de recuperação quanto no percentual de CCOs de graus I e II (Quadro 2). Ainda no Quadro 2, observa-se que o percentual de oócitos parcialmente desnudos/desnudos/degenerados foi novamente maior no grupo NP4, independentemente do grau de maturidade sexual, sendo de 20,7\% para nulíparas e de 17,2\% para multíparas em comparação ao grupo P4 (13,3\% e 15,4\%, respectivamente). Os CCOs expandidos foram percentualmente superiores nas multíparas P4 (11,7\%), seguindo a mesma tendência já apresentada e discutida no Quadro 1.

Conforme apresentado no Quadro 3, observa-se que folículos pequenos possuem uma menor taxa de recuperação em relação aos grandes e médios $(33,2 \%, 72,0 \%$ e $65,5 \%$, respectivamente $(\mathrm{P}<0,05)$, porém o percentual de CCOs, potencialmente selecionados para PIV ou maturáveis (Graus I e II), foi maior em folículos pequenos e médios $(57,4 \%$ e $59,3 \%$, respectivamente $)(\mathrm{P}<0,05)$. 0 contrário foi observado na quantidade de oócitos parcialmente desnudos, desnudos e degenerados e CCOs expandidos que foi significativamente maior nos folículos grandes $(23,9 \%$ e $14,4 \%$, respectivamente).

\section{DISCUSSÃ0}

Resultados similares foram encontrados em outras publicações, nas quais foram também utilizados ovários de matadouro, não tendo sido encontrada diferença na qualidade dos oócitos entre animais jovens (a partir de 18 me- ses) e senis (até 17 anos), embora tenham notado redução na produção folículos e gametas nos animais mais velhos (Katska \& Smorag 1984). Em fêmeas bovinas, o aumento da idade, sobretudo após a fase de declínio reprodutivo senescente, está associado com a elevação na concentração de gonadotropinas, em razão da redução no potencial esteroidogênico das células foliculares ovarianas (Malhi et al. 2005). Estes distúrbios endócrinos, transitórios ou não, são causas comuns de perda na competência oocitária (Torres-Júnior et al. 2008).

Além disso, estudos recentes têm consolidado a informação de que, apesar das variações individuais, há alta repetibilidade no número de folículos recrutados em cada onda de crescimento folicular, independentemente da idade, raça, país de origem ou estação do ano (Mossa et al. 2012).

Em um recente trabalho realizado com fêmeas cíclicas, Nasser et al. (2011) verificaram que, para iniciar a superovulação durante primeira onda de crescimento folicular pós ovulação, é necessário suplementar as fêmeas com progesterona exógena, caso contrário não há expansão das células do cumulus e maturação oocitária em resposta à administração de LH exógeno, culminando com baixa qualidade embrionária. Além disso, o efeito da suplementação com progesterona durante o desenvolvimento do folículo pré-ovulatório potencializa o estabelecimento do corpo lúteo, aumentando consequentemente a concentração de progesterona circulante durante o desenvolvimento embrionário inicial e incrementando de forma particular a elongação do embrião em fase de pré-implatação (Lonergan \& Forde 2014, Maillo et al. 2014, Wiltbank et al. 2014). 
Quadro 3. Efeitos do diâmetro folicular sobre a taxa de recuperação e qualidade de CCOs

\begin{tabular}{lccc}
\hline \multicolumn{1}{c}{ Variável } & $\begin{array}{c}\text { Pequenos }(<6 \mathrm{~mm}) \\
\text { no./total }(\%)\end{array}$ & $\begin{array}{c}\text { Médios }(6 \mathrm{a} 9 \mathrm{~mm}) \\
\text { no./total }(\%)\end{array}$ & $\begin{array}{c}\text { Grandes (>9mm) } \\
\text { no./total (\%) }\end{array}$ \\
\hline Taxa Recuperação & $1250 / 3767(33,2)^{\mathrm{b}}$ & $605 / 923(65,5)^{\mathrm{a}}$ & $180 / 250(72,0)^{\mathrm{a}}$ \\
Grau I & $717 / 1250(57,4)^{\mathrm{a}}$ & $359 / 605(59,3)^{\mathrm{a}}$ & $83 / 180(46,1)^{\mathrm{b}}$ \\
Grau II & $207 / 1250(16,6)$ & $122 / 605(20,2)$ & $28 / 180(15,6)$ \\
Parcialmente desnudos/ & $214 / 1250(17,1)^{\mathrm{b}}$ & $76 / 605(12,6)^{\mathrm{c}}$ & $43 / 180(23,9)^{\mathrm{a}}$ \\
Desnudos / Degenerados & & & \\
Expandidos & $112 / 1250(9,0)^{\mathrm{b}}$ & $48 / 605(7,9) \mathrm{b}$ & $26 / 180(14,4)^{\mathrm{a}}$
\end{tabular}

$\overline{\text { abc Letras diferentes na mesma linha }(\mathrm{P}}<0,05)$; teste de Qui-quadrado.

Ainda, tem sido reportado que o intercâmbio entre o oócito e as células do cumulus é fator chave para o sucesso na maturação, fertilização e início de desenvolvimento embrionário, o que coloca o estudo das células do cumulus como prioritário para predizer a competência oocitária (Tanghe et al. 2002). Adicionalmente a manutenção de um acoplamento funcional adequado entre oócito e cúmulus parece ser crucial para sustentar uma ordenada condensação da cromatina in vitro (Dieci et al. 2013, Lodde et al. 2013, Luciano et al. 2014). Substâncias regulatórias produzidas pelo oócito têm importantes funções no metabolismo das células do cúmulos e os produtos dessas células somáticas participam ativamente do crescimento e maturação de oócitos (Landim-Alvarenga \& Maziero 2014).

Segundo Nasser (2011), somente em fêmeas que apresentam equilíbrio no balanço endócrino de P4 e LH, esta associação proporciona sincronização da maturação nuclear oocitária e esteroidogênese, produzindo oócitos e embriões de maior qualidade. Neste sentido, justifica-se a resposta encontrada no presente estudo, sinalizada pelo maior percentual de CCOs expandidos e, portanto, em fase final de maturação em fêmeas com P4, ao contrário de fêmeas sem $\mathrm{P} 4$, ou em anestro, onde se encontrou maior percentual de CCOs efetivamente de baixa qualidade.

O maior percentual de CCOs maturados (cumulus expandido) das fêmeas com progesterona pode ser devido ao efeito fisiológico da relação progesterona:estradiol no processo de reativação da meiose oocitária LH-dependente (Roth \& Hansen 2005). Segundo Wiltbank et al. (2006), nos casos de anestro fisiológico, nutricional ou metabólico, a diminuição nas concentrações circulantes de esteróides sexuais retarda e diminui a amplitude do pico pré-ovulatório de LH, comprometendo a maturação e a viabilidade do CCOs ovulado.

Considerando que folículos em fase final de maturação geralmente apresentam células do cumulus expandidas e descompactadas (Assey et al. 1994), era de se esperar que em folículos grandes $(>9 \mathrm{~mm})$ realmente se encontrasse maior percentual de CCOs expandidos ou parcialmente desnudos, diminuindo, consequentemente, o total de estruturas Grau I e II.

Segundo Hendriksen et al. (2004), a proporção de cumulus expandidos foi maior em folículos com oito dias pós emergência. Similarmente, houve interação entre a presença do folículo dominante e a alta ocorrência de oócitos desnudos também no ovário contralateral (Vassena et al. 2003), reafirmando a importância do folículo dominante na estabilização, atresia e baixa qualidade oocitária dos folículos subordinados.

A influência do tamanho folicular na competência oocitária para o desenvolvimento in vitro também foi comprovada por Lonergan et al. (1994), porém, estes verificaram aumento significativo na produção de embriões oriundos de oócitos aspirados de folículos com mais de $6 \mathrm{~mm}$ de diâmetro. Os mesmos autores também observaram a menor recuperação de oócitos quando havia predomínio de grandes folículos no momento da aspiração, e com isso constataram taxa de recuperação maior em folículos entre $2 \mathrm{e}$ $6 \mathrm{~mm}$. Neste sentido, a presença de folículos dominantes interfere tanto no número quanto na viabilidade dos folículos disponíveis para superovulação (Bungartz \& Niemann 1994) e também para aspiração folicular (Torres-Júnior et al. 2006b).

Essa relação entre a taxa de recuperação e as consequentes alterações morfológicas no complexo cumulus oócito (Bols et al. 1998), podem ainda ser atribuídas aos próprios folículos, como viscosidade do fluido folicular (Goodhand et al. 1999), quantidade de material a ser aspirado e pressão intrafolicular (Seneda 1999), assim como a aderência do oócito na membrana do folículo, ou mesmo inerentes à mecânica da OPU, diâmetro da agulha, pressão de aspiração folicular, além da experiência e destreza do técnico, entre outros (Viana et al. 2003).

Finalmente, estes achados resultam em novas informações acerca das interações multifatoriais que interferem na qualidade de CCOs bovinos, com a perspectiva de contribuir com a aplicação prática da OPU-PIV.

\section{CONCLUSÃO}

A categoria da doadora e a progesterona não influenciaram a qualidade de CCOs selecionados para PIV e, folículos menores apresentam CCOs de melhor qualidade.

Agradecimentos.- Os autores agradecem à Fundação de Amparo à Pesquisa e ao Desenvolvimento Tecnológico do Maranhão (FAPEMA; processo APP-Universal 01040/09) pelo aporte financeiro da pesquisa e à bolsa concedida ao primeiro autor.

\section{REFERÊNCIAS}

Assey R.J., Hyttel P., Greve T. \& Purwantara B. 1994. Oocyte morphology in dominant and subordinate follicles. Mol. Reprod. Dev. 37:335-344.

Basso V.T., Nascimento P.P. \& Castilho C. 2006. Efeito do diâmetro folicular sobre a qualidade dos oócitos obtidos de ovários de fêmeas bovinas de abatedouro. Colloquium Agrariae 2(1):12-16. 
Blondin P. \& Sirard M. 1995. Oocyte and follicular morphology as determining characteristics for developmental competence in sheep oocytes. J. Reprod. Fertil. 62:575-582.

Bungartz L. \& Niemann H. 1994. Assesment of the presence of a dominant follicle and selection of dairy cows suitable for superovulation by a single ultrasound examination. J. Reprod. Fertil. 101:583-591.

Costa E.P. 1994. Aspectos morfológicos, citológicos, ultra-estruturais e desenvolvimento de ovócitos bovinos in vitro. Tese de Doutorado, Escola de Veterinária, Universidade Federal de Minas Gerais, Belo Horizonte, MG. 155p.

Dieci C., Lodde V., Franciosi F., Lagutina I., Tessaro I., Modina S.C., Albertini D.F., Lazzari G., Galli C. \& Luciano A.M. 2013. The effect of cilostamide on gap junction communication dynamics, chromatin remodeling, and competence acquisition in pig oocytes following parthenogenetic activation and nuclear transfer. Biol. Reprod. 89:68.

Fatehi A.N., Roelen B.A., Colenbrander B., Schoevers E.J., Gadella B.M., Beverst M.M. \& Van Der Hurk R. 2005. Presence of cumulus cells during in vitro fertilization protects the bovine oocyte against oxidative stress and improves first cleavage but does not affect further development. Zygote 13(2):177-185.

Galli C., Crotti G., Notari C., Turini P., Duchi R. \& Lazzari G. 2001. Embryo production by ovum pick up from live donors. Theriogenology 55:13411357.

Gimenes L.U., Ferraz M.L., Almeida R.G., Dias F.B.R., Pereira F.M.M., Araújo A.B., Joaquim D.C., Watanabe Y., Meirelles F.V., Beltran M.P., Pinheiro D.M., Nogueira G.P., Torres-Júnior J.R.S., Ayres H. \& Baruselli P.S. 2007a. Efeito do dia de aspiração folicular em relação à emergência $(2,4$ ou 6 dias) sobre a qualidade de embriões bovinos produzidos in vitro (Parte 1). XXI Reunião da Sociedade Brasileira de Tecnologia de Embriões. Acta Scientiae Veterinariae 35:1170.

Gimenes L.U., Ferraz M.L., Almeida R.G., Dias F.B.R., Pereira F.M.M., Araújo A.B., Joaquim D.C., Watanabe Y., Meirelles F.V., Torres-Júnior J.R.S., Ayres H., Madureira E.H. \& Baruselli P.S. 2007b. Efeito do dia de aspiração folicular em relação à emergência (2, 4 ou 6 dias) sobre a qualidade de embriões bovinos produzidos in vitro (Parte 2). XXI Reunião da Sociedade Brasileira de Tecnologia de Embriões. Acta Scientiae Veterinariae 35:1171.

Gopichandran N. \& Leese H.J. 2006. The effect of paracrine/autocrine interactions on the in vitro culture of bovine pre-implantation embryos. Reproduction 131:269-277.

Hendriksen P.J.M., Steenweg W.N.M., Harkema J.C., Merton J.S., Bevers M.M., Vos P.L.A.M. \& Dieleman S.J. 2004. Effect of different stages of the follicular wave on in vitro developmental competence of bovine oocytes. Theriogenology 61:909-920.

International Embryo Transfer Society (IETS). 2013. Embryo Transfer Newsletter. Available on <http//www.iets.org> Accessed on June 1, 2014.

Katska L. \& Smorag Z. 1984. Number and quality of oocytes in relation to age of cattle. Anim. Reprod. Sci. 7:451-460.

Landim-Alvarenga F.C. \& Maziero R.R.D. 2014. Control of oocyte maturation. Anim. Reprod. 11(3):150-158.

Lodde V., Franciosi F., Tessaro I., Modina S.C. \& Luciano A.M. 2013. Role of Gap junction-mediated communications in regulating large-scale chromatin configuration remodeling and embryonic developmental competence acquisition in fully grown bovine oocyte. J. Assist. Reprod. Genet. 30:1219-1226.

Lonergan P., Monaghan P., Rizos D., Boland M.P. \& Gordon I. 1994. Effect of follicle size on bovine oocyte quality and developmental competence following maturation, fertilization, and culture in vitro. Mol. Reprod. Dev. 37:48-53.

Lonergan P., Rizos D., Kanka J., Nemcova L., Mbaye A.M. \& Kingston M. 2003. Temporal sensitivity of bovine embryos to culture environment after fertilization and the implications for blastocyst quality. Reproduction 126:337-346.

Lonergan P. \& Forde N. 2014. Maternal-embryo interaction leading up to the initiation of implantation of pregnancy in cattle. Animal. 8 (1):64-69.

Luciano A.M., Franciosi F., Dieci C., Tessaro I., Terzaghi L., Modina S.C. \& Lodde V. 2014. Large-scale chromatin structure and function changes during oogenesis: the interplay between oocyte and companion cumulus cells. Animal Reprod. 11(3):141-149.

Maillo V., Duffy P., O`Hara L., de Frutos C., Kelly A.K., Lonergan P. \& Rizos D. 2014. Effect of hCG administration during corpus luteum establishment on subsequent corpus luteum development and circulating progesterone concentrations in beef heifers. Reprod. Fertil. Dev. 26:367-374.

Malhi P.S., Adams G.P. \& Singh J. 2005. Bovine model for the study of reproductive aging in women: follicular, luteal and endocrine characteristics. Biol. Reprod. 73:45-53.

Mapletoft J.R. 2013. History and perspectives on bovines embryo transfer. Anim. Reprod. 10(3):168-173.

Mossa F., Walsh S.W., Butler S.T., Berry D.P., Carter F., Lonergan P., Smith G.W., Ireland J.J. \& Evans A.C. 2012. Low numbers of ovarian follicles $>3 \mathrm{~mm}$ in diameter are associated with low fertility in dairy cows. J. Dairy Sci. 95:2355-2361.

Nasser L.F., Sa M.F., Reis E.L., Rezende C.R., Mapletoft R.J., Bo G.A. \& Baruselli P.S. 2011. Exogenous progesterone enhances ova and embryo quality following superstimulation of the first follicular wave in Nelore (Bos indicus) donors. Theriogenology 76:320-327.

Pontes J.H., Melo Sterza F.A., Basso A.C., Ferreira C.R., Sanches B.V., Rubin K.C. \& Seneda M.M. 2011. Ovum pick up, in vitro embryo production, and pregnancy rates from a large-scale commercial program using $\mathrm{Ne}-$ lore cattle (Bos indicus) donors. Theriogenology 75:1640-1646.

Roth Z. \& Hansen P.J. 2005. Disruption of nuclear maturation and rearrangement of cytoskeletal elements in bovine oocytes exposed to heat shock during maturation. Reproduction 129:235-244.

Sampaio I.B.M. 2002. Estatística Aplicada à Experimentação Animal. 2ª ed. FEP-MVZ, Belo Horizonte. 265p.

Stroud B. 2012. The year 2011 worldwide statistics of embryo transfer in domestic farm animals. IETS Newslettter 50:16-25.

Tanghe S., Van Soom A., Nauwynck H., Coryn M. \& De Kruif A. 2002. Functions of the cumulus oophorus during oocyte maturation, ovulation, and fertilization: a minireview. Mol. Reprod. Dev. 61:414-424.

Torres-Júnior J.R.S., Pires M.F.A., Sá W.F., Ferreira A.M., Viana J.H.M., Camargo L.S.A., Ramos A.A., Folhadella I.M., Polisseni J., Freitas C., Clemente C.A.A., Sá Filho M.F., Souza A.H. \& Baruselli P.S. 2006a. Efeito da presença do corpo lúteo no momento da aspiração folicular sobre a recuperação e competência in vitro de oócitos (Bos indicus). XX Reunião Anual da Sociedade Brasileira de Tecnologia de Embriões. Acta Scientiae Veterinariae 34:472-472.

Torres-Júnior J.R.S., Pires M.F.A., Sá W. F., Ferreira A.M., Viana J.H.M., Camargo L.S.A., Ramos A.A., Folhadella I.M., Polisseni J., Freitas C., Clemente C.A.A., Sá Filho M.F., Souza A.H., Martins C.M. \& Baruselli P.S. 2006b. Efeito da codominância de folículos no momento da aspiração folicular sobre a recuperação e competência in vitro de oócitos (Bos indicus). XX Reunião Anual da Sociedade Brasileira de Tecnologia de Embriões. Acta Scientiae Veterinariae 34:473-473.

Torres-Júnior J.R.S., Pires M.F.A., Sá W.F. Ferreira A.M., Viana J.H.M., Camargo L.S.A., Ramos A.A., Folhadella I.M., Polisseni J., Freitas C., Clemente C.A.A., Sá Filho M.F., Paula-Lopes F.F. \& Baruselli P.S. 2008. Effect of maternal heat-stress on folicular growth and oocyte competence in Bos indicus cattle. Theriogenology 69:155-166.

Vassena R., Mapletoft R.J., Allodi S. Singh J. \& Adams G.P. 2003. Morphology and developmental competence of bovine oocytes relative to follicular status. Theriogenology 60:923-932.

Viana J.H.M., Nascimento A.A., Pinheiros N.L., Ferreira A.M., Camargo L.S.A., Sá W.F. \& Marques Júnior A.P. 2003. Caracterização de sequelas subsequentes à punção folicular em bovinos. Pesq. Vet. Bras. 23(3):119-124.

Viana J.H.M., Siqueira L.G.B., Palhão M.P. \& Camargo L.S.A. 2010. Use of in vitro Fertilization Technique in the Last Decade and its Effect on Brazilian Embryo Industry and Animal Production. Acta Scientiae Veterinariae 38(Suppl.2):661-674.

Wiltbank M.C., Lopez H., Sartori R., Sangsritavong S. \& Gumen A. 2006. Changes in reproductive physiology of lactating dairy cows due to elevated steroid metabolism. Theriogenology 65:17-29.

Wiltbank M.C., Souza A.H., Carvalho P.A., Cunha A.P., Giordano J.O., Fricke P.M., Baez G.M. \& Diskin M.G. 2014. Phisiological and practical effects of progesterone on reproduction in dairy cattle. Animal. 8(1):70-81. 